Association for Information Systems

AIS Electronic Library (AISeL)

Wirtschaftsinformatik 2021 Proceedings

Track 17: Digital transformation \& business

models

\title{
Untangling the Open Data Value Paradox: How Organizations Benefit from Revealing Data
}

Tobias Enders

Karlsruher Institut für Technologie

Carina Benz

Karlsruher Institut für Technologie

Gerhard Satzger

Karlsruher Institut für Technologie

Follow this and additional works at: https://aisel.aisnet.org/wi2021

Enders, Tobias; Benz, Carina; and Satzger, Gerhard, "Untangling the Open Data Value Paradox: How Organizations Benefit from Revealing Data" (2021). Wirtschaftsinformatik 2021 Proceedings. 3. https://aisel.aisnet.org/wi2021/HDigitaltransformation17/Track17/3

This material is brought to you by the Wirtschaftsinformatik at AIS Electronic Library (AISeL). It has been accepted for inclusion in Wirtschaftsinformatik 2021 Proceedings by an authorized administrator of AIS Electronic Library (AISeL). For more information, please contact elibrary@aisnet.org. 


\title{
Untangling the Open Data Value Paradox: How Organizations Benefit from Revealing Data
}

\author{
Tobias Enders, Carina Benz, and Gerhard Satzger \\ Karlsruhe Institute of Technology, IISM and KSRI, Karlsruhe, Germany \\ \{tobias.enders, carina.benz, gerhard.satzger\}@kit.edu
}

\begin{abstract}
Inspired by governmental institutions publishing data for more than a decade, also private sector organizations have started engaging in open data initiatives in recent years. While monetary expenses to engage in open data are tangible, its benefits remain vague, thus fueling the open data paradox. We conduct a set of expert interviews to untangle this paradox to elicit potential benefits that may originate from engaging in open data in the private sector. Our preliminary results show three distinct groups of benefits: internal improvements, innovation driver, and external visibility. With this paper, we lay the foundation for a comprehensive model on exploring open data benefits. For practitioners, we showcase a novel path to extract value from data and to monetize it.
\end{abstract}

Keywords: open data, benefits, data value, data monetization

\section{Introduction}

By now, most organizations acknowledge the role of data as a strategic asset that carries implicit value [1]. Inspired by contemporary trends towards openness and social responsibility, firms let their organizational boundaries become permeable to enable collaboration and innovation [2]. In recent years, private sector firms have started sharing their data with the general public as open data. Given a high level of uncertainty of its benefits, investments are still scarce and fuel the open data paradox [3].

The paradox describes a setting between data provider and data consumer: While data consumers refrain from building services or business models based on open data given its uncertain perpetuity, data providers hesitate to make investments given a lack of evidence of innovation and added value [3]. This paper aims to untangle one side of the paradox by showcasing benefits and thus calling for more investments into open data. Our overall objective of this study is the development of a comprehensive model on the benefits of open data in private sector organizations. This paper represents the first pillar, which is based upon expert interviews.

Given that extant literature offers little insight into benefits of engaging in open data in private sector organizations, our initial data collection is based upon a set of expert interviews. This approach allows obtaining first-hand knowledge from firms that have been active in open data. Hence, we pursue the following research questions: "What are the benefits from engaging in open data in private sector organizations?" To 
address this question, we conduct ten semi-structured interviews across multiple industry sectors. Preliminary results show that there are three distinct groups of benefits: internal improvements, innovation driver, and external visibility.

The paper is structured as follows: Section 2 introduces the fundamentals of open data followed by the research design in section 3 . Section 4 discusses the preliminary results of our study before closing with a conclusion and research outlook in section 5 .

\section{Fundamentals of Open Data}

Over the years, literature has produced various understandings and conceptualizations of what is meant by openness [4]. Open data - representing an open resource in the universe of openness - makes no exception: A multitude of definition of what open data entails has emerged. We follow the concept that defines open data as a form of content that can be freely used, modified, and shared by anyone and for any purpose [5]. Despite this notion, organizations oftentimes restrict data re-use and re-distribution and thereby dilute the meaning of data being truly open.

Given the ambiguity in research on "openness", it is crucial to contextualize open data towards adjacent research fields. Schlagwein et al. [6] suggest a framework to conceptualize different research streams: Openness as higher order concept, open sources, open process and open effects. In line with the proposed framework, we understand open data to be an open resource similar to open API [7] and open source software [8]. In contrast, open processes include open innovation [2, 9] and crowdsourcing [10]. While open resources may represent an input to open processes, we must clearly distinguish between those fields of research and their contributions.

While the phenomenon of open data is just gaining momentum in the private sector [11], open government data (OGD) has already proven to enable value creation [12]. Driven by changes in legislation, such as in the European Union [13] and Unites States [14], the public sector has been a role model on open data initiatives for almost two decades. Whilst research into expected benefits of OGD provides initial insights, little is known about potential benefits in the private sector. As for OGD, institutions strive, for instance, for a higher level of transparency $[15,16]$, democratic accountability and improved citizen services [17]. Furthermore, there is a hope that open data promotes collaboration activities between citizens and government [18, 19]. From an economic perspective, OGD has also proven to enable the formation of new business models [20]. Given that public and private organizations operate towards different objectives maximization of social welfare versus maximization of profits -, additional research is needed to capture and contrast those benefits.

\section{Research Design}

To explore benefits derived from open data, we conduct a set of semi-structured expert interviews across multiple industry sectors [21]. For the selection of interview participants, we follow a criterion-i purposeful sampling approach [22]. The criteria are defined as such that all interviewees must have direct knowledge of working with open 
data in private sector organizations, hence, provide first-hand information. Both, technical and business roles of the interviewees are in focus to explore different perspectives. An overview of the experts is provided in Table 1. In total, ten interviews are conducted; either over the phone or in-person. Each interview lasts between 45 minutes and one hour and is recorded and transcribed for further analysis.

The interviews are analyzed by conducting two distinct coding cycles [23]. To account for the explorative nature of the study, an open coding cycle with no predefined list of codes is used for the first iteration. The second coding cycle consists of an axial coding to resemble codes and to ensure that categories and subcategories relate to each other accordingly. To verify the results, we ask a second, independent researcher to re-code all interviews. Discrepancies between the coding of the researchers are discussed until a mutual agreement is reached. The software MAXQDA supports this work.

Table 1. Overview of interviewees

\begin{tabular}{lll}
\hline Interviewee & Job Role & Industry \\
\hline 1 & Team Lead IT \& Engineering & Chemicals \\
2 & Chief Data Officer & Energy \\
3 & Head Big Data Strategy & Energy \\
4 & Chief Data Officer & Banking \\
5 & Head Data-Based Business Models & Automotive \\
6 & Head Data Strategy & Automotive \\
7 & IT Architect & Travel \& Transport \\
8 & IT Architect & Travel \& Transport \\
9 & Business Data Manager & Logistics \\
10 & IT Enterprise Architect & Travel \& Transport \\
\hline
\end{tabular}

\section{$4 \quad$ Preliminary Results}

Uncertainty about open data benefits keeps organizations from investing in these initiatives. Our preliminary results show that firms experience benefits across multiple domains: Open data contributes to internal improvements, acts as an innovation driver and improves the firm's visibility towards external stakeholders. A visual representation is shown in Figure 1 followed by an explanation of each category.
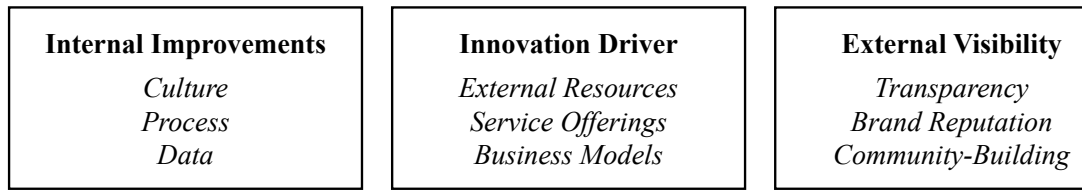

Figure 1. Open data benefits

Internal Improvements. Despite open data being an initiative primarily targeting external stakeholders, it offers multiple internal benefits to the data provider itself. We 
separate those benefits into culture, process and data. We observe that the workforce (culture) experiences a mindset shift towards a sharing and collaborative behavior. Driven by the role model of sharing data with external parties, there is an increased awareness of employees to value and foster sharing activities within the organization itself. Furthermore, employees are intrinsically motivated to acquire new skills on data management, which can be transferred to subsequent projects. The IT Architect of Travel \& Transport company recalls: "(...) the employees from our department that participated [in the open data project] learnt a lot, especially how to handle the data". As for processes, we observe that open data supports efforts to standardize processes across the organization. For instance, access management processes are streamlined across departments to enable all employees to have access to a common data repository. Also, having access to a structured open data interface expedites internal software development cycles, which directly translates into cost savings. In a particular case, a travel \& transport firm shortened their application development cycle times by up to $50 \%$. As for a data focus, we find that engaging in open data leads to the creation of central data repositories (e.g., data lake), which serve as a one-stop data shop for the entire organization. Also, open data promotes automation efforts given pre-curated data for automated report generation. In summary, we observe that open data contributes to a de-silofication across the organizations; i.e., organizational silos fade away in favor of shared repositories, processes and mindsets.

Innovation Driver. Revealing data to the outside can reinforce a company's innovation potential. It offers access to external resources and reflects an option to combat internal capacity constrains: Through open data, companies can profit from time, knowledge, skills and ideas of externals engaging with the data sets. Being intrinsically motivated, external problem solvers invest their personal resources free of charge and contribute to idea generation and problem solving. This creative potential may even translate into new service offerings being implemented by the organization itself (e.g., through IP purchasing or licensing) or complementing their business when being offered by the external innovator. In fact, we find external innovators to develop solutions to previously unknown problems, e.g. by addressing issues of minority customer groups. In a concrete use case, a train and rail operator made information about available cargo spaces on their passenger trains available as open data. Inspired by the idea to reduce $\mathrm{CO}_{2}$ emissions for transporting parcels, a Swiss-based startup used this data to develop a service that allowed same-day parcel delivery by train. For the last mile, the startup uses cargo bikes. Through the use of open data, a win-win situation for both sides emerged: For the startup, the data enabled the service and hence their business model as a whole. For the train \& rail operator, additional cargo transported meant additional revenue. Interestingly, open data can enable new business models for the data provider as well: While open data is revealed free of charge, organizations start implementing freemium payment models. We find that firms allow access to limited or aggregated open data as a trigger for interested parties to pay for more granular data. Thus, companies establish new business models and thereby monetize their data assets. While extant literature discusses the emergence of new business models in connection with open government data [12], it represents a novel perspective for private sector organizations. In summary, we observe open data to spark innovation by leveraging 
resources outside the company and opening potential for novel business models and services.

External Visibility. Open data offers benefits related to an enhanced external visibility of the firm. Publishing data creates transparency by allowing insights into the company's operations. As a consequence, the interviewees confirm a positive impact on the brands' reputation. This is further accelerated by press releases, spreading the word via online and offline media such as blogs, business journals and newspapers. We find that this leads to positive outcomes. For instance, firms experience a spike in the number of applicants for open job positions - especially from recent university graduates. The Chief Data Officer from an Energy company notes: "[open data] is an opportunity for us to present the company and get to know university graduates (...) that we might have the opportunity to hire at a later point in time". Similarly, other external entities take notice of the company, such as investors in pursuit of new investment opportunities. Moreover, we observe community-building activities between the firm, creative minds and developers. Establishing a relationship with the community is of utmost importance to enable feedback mechanisms and learn about data needs. Summarizing, open data enhances transparency, brand reputation and community-building, resulting in improved visibility towards external stakeholders.

\section{Conclusion and Outlook}

This paper investigates how private sector organizations engaging in open data benefit from these initiatives. As a first step towards a holistic model on open data benefits, we conduct expert interviews to reveal insights on the companies' experience. We find open data to contribute to internal improvements, to act as an innovation driver and to enhance the firm's visibility towards external stakeholders. For organizations, we illustrate that engaging in open data represents a novel path to extract value from data and to benefit in monetary and non-monetary ways. By providing evidence of value created through open data, we disentangle one side of the open data value paradox. Most notably, open data creates an opportunity for firms to obtain access to skills, time and ideas at no additional charge to co-create value and boost innovation.

While this study's findings are limited given that only single observations were made for each of the organizations, we plan on extending our knowledge base to arrive at a comprehensive model of open data benefits. In particular, we aim to deepen our insights by drawing on in-depth case studies with firms that have been practicing open data for several years. This allows us to observe benefits as they evolve over a longer period of time and hence, we are not dependent on retrospective considerations of individuals. Those findings will be complemented by and contrasted with a structured literature review on open data benefits across multiple domains. In addition to extending our data base, we intend to further analyze organizational change implications of engaging in open data. For instance, open data enables new business models that need to be aligned and integrated with existing ones. While this work has a strong focus on potential benefits of open data, future research needs to critically reflect on the drawbacks and risks that may occur when revealing open data in a digital ecosystem. 


\section{References}

1. Wixom, B.H., Ross, J.W.: How to monetize your data? MIT Sloan Manag. Rev. 58, (2017).

2. Chesbrough, H.: The future of open innovation. Res. Manag. 60, 35-38 (2017).

3. Jetzek, T.: Innovation in the Open Data Ecosystem: Exploring the Role of Real Options Thinking and Multi-sided Platforms for Sustainable Value Generation through Open Data. In: Analytics, Innovation, and Excellence-Driven Enterprise Sustainability. pp. 137-168. Palgrave Macmillan, New York (2017).

4. Dahlander, L., Gann, D.M.: How open is innovation? Res. Policy. 39, 699-709 (2010).

5. Open Knowledge Foundation: The Open Definition, https://opendefinition.org/ (Accessed: 01.07.2020).

6. Schlagwein, D., Conboy, K., Feller, J., Leimeister, J.M., Morgan, L.: "Openness” with and without Information Technology: A framework and a brief history. J. Inf. Technol. 32, 297-305 (2017).

7. Benlian, A., Hilkert, D., Hess, T.: How open is this Platform? The meaning and measurement of platform openness from the complementers' perspective. J. Inf. Technol. 30, 209-228 (2015).

8. Henkel, J.: Selective revealing in open innovation processes: The case of embedded Linux. Res. Policy. 35, 953-969 (2006).

9. West, J., Salter, A., Vanhaverbeke, W., Chesbrough, H.: Open Innovation: The Next Decade. Res. Policy. 43, 805-811 (2014).

10. Howe, J.: Crowdsourcing: How the power of the crowd is driving the future of business. Random House (2008).

11. Enders, T., Benz, C., Schüritz, R., Lujan, P.: How To Implement an Open Data Strategy? Analyzing Organizational Change Processes To Enable Value Creation by Revealing Data. In: Proceedings of 28th European Conference on Information Systems (2020).

12. Ahmadi Zeleti, F., Ojo, A., Curry, E.: Exploring the economic value of open government data. Gov. Inf. Q. 33, 535-551 (2016).

13. European Parliament: Directive 2003/98/EC on the re-use of public sector information, https://eur-lex.europa.eu/LexUriServ/LexUriServ.do?uri=OJ:L:2003:345:0090:0096: en:PDF (Accessed: 05.08.2020).

14. Obama, B.: Transparency and Open Government, https://obamawhitehouse.archives.gov/ the-press-office/transparency-and-open-government (Accessed: 15.05.2020).

15. Bertot, J.C., Jaeger, P.T., Grimes, J.M.: Using ICTs to create a culture of transparency: Egovernment and social media as openness and anti-corruption tools for societies. Gov. Inf. Q. 27, 264-271 (2010).

16. McDermott, P.: Building open government. Gov. Inf. Q. 27, 401-413 (2010).

17. Janssen, M., Charalabidis, Y., Zuiderwijk, A.: Benefits, Adoption Barriers and Myths of Open Data and Open Government. Inf. Syst. Manag. 29, 258-268 (2012).

18. Kassen, M.: A promising phenomenon of open data: A case study of the Chicago open data project. Gov. Inf. Q. 30, 508-513 (2013).

19. Johnson, P., Robinson, P.: Civic Hackathons: Innovation, Procurement, or Civic Engagement? Rev. Policy Res. 31, 349-357 (2014).

20. Janssen, M., Zuiderwijk, A.: Infomediary Business Models for Connecting Open Data Providers and Users. Soc. Sci. Comput. Rev. 32, 694-711 (2014). 
21. King, N.: Using interviews in qualitative research. In: Cassell, C. and Symon, G. (eds.) Essential guide to qualitative methods in organizational research. pp. 11-22. SAGE (2004). 22. Palinkas, L.A., Horwitz, S.M., Green, C.A., Wisdom, J.P., Duan, N., Hoagwood, K.: Purposeful Sampling for Qualitative Data Collection and Analysis in Mixed Method Implementation Research. Adm. Policy Ment. Heal. Res. 42, 533-544 (2015).

23. Saldaña, J.: The Coding Manual for Qualitative Researchers. SAGE, London (2009). 